\title{
Molecular Characterization of Extended Spectrum $\beta$-lactamase and Carbapenemase Producing Klebsiella pneumoniae from a Tertiary Care Hospital
}

\author{
Beena Hosdurg Bhaskar ${ }^{1}$, Shalini Shenoy Mulki ${ }^{2}$, Sangeetha Joshi ${ }^{3}$, Ranjeeta Adhikary ${ }^{4}$, Bhavana Malavalli Venkatesh ${ }^{5}$
}

\begin{abstract}
Objective: The extended-spectrum beta-lactamase (ESBL) and carbapenemase producing gram-negative bacteria among the members of Enterobacteriaceae are of major health concern globally. The present study was carried out to determine proportion and genetic characterization of ESBL and carbapenemase producing Klebsiella pneumoniae strains isolated from intensive care units of a tertiary care hospital.

Materials and methods: A total of 250 non-duplicate K. pneumoniae isolates were recovered from various clinical specimens from our intensive care units from May 2014 to May 2015. Antibiotic susceptibility testing was performed as recommended by Clinical and Laboratory Standard Institute. Phenotypic identification of ESBL and carbapenemase producing isolates were confirmed by the double-disk synergy test, modified Hodge test, imipenem and imipenem-EDTA combined test, respectively. Molecular characterization of $\beta$-lactamase genes were performed by polymerase chain reaction.

Results: Out of 250 Klebsiella pneumonaie, $84 \%$ isolates were ESBL producers, $66 \%$ were carbapenem resistant based on their reduced susceptibility to imipenem, meropenem and ertapenem. Among these 165 carbapenem resistant isolates, $9.7 \%$ were positive for $b / a_{\mathrm{NDM}-1}$ and these isolates were also found to be positive for one or more bla genes. Co-carriage of AmpC in ESBL and carbapenem resistant isolates were $7.8 \%$ and $3.6 \%$, respectively and were negative for $b / a_{\mathrm{KPC}}$ genes.

Conclusion: The study indicated the prevalence of ESBLs and $b / a_{\mathrm{NDM}-1}$, with additional bla genes and AmpC among the $K$. pneumoniae isolates in our intensive care units. NDM-1 producing Enterobacteriaceae is a growing health care problem. Detection of the prevalence of antibacterial resistance pattern helps towards improved antibiotic policy and empirical antibiotic treatment.

Keywords: AmpC, Carbapenemase, Extended spectrum $\beta$-lactamase, Metallo $\beta$-lactamase

Indian Journal of Critical Care Medicine (2019): 10.5005/jp-journals-10071-23118
\end{abstract}

\section{INTRODUCTION}

nfections due to multidrug resistant (MDR) Enterobacteriaceae are an important cause of morbidity and mortality worldwide. Carbapenems are most often used in this treatment. The emergence of resistance to these agents has become a serious health concern globally ${ }^{1}$.

Klebsiella pneumoniae is one of the most common Gramnegative bacteria showing resistance to multiple antibiotics. The development of extended-spectrum cephalosporins in the early 1980 s was regarded as a major addition to our therapeutic armamentarium in the fight against beta-lactamase mediated bacterial resistance. The emergence of enzymes that have the ability to hydrolyze this cephalosporin's seriously compromised the efficacy of these lifesaving antibiotics. These enzymes were called extended spectrum beta lactamases ${ }^{2}$. Extended spectrum beta-lactamases are plasmid-mediated enzymes that are capable of conferring bacterial resistance to the penicillins, first, second third, fourth generation cephalosporins and aztreonam.

They do this by hydrolysis of these antibiotics but they are inhibited in vitro by beta- lactamase inhibitors ${ }^{3}$.

ESBL is predominantly found in Klebsiella spp. and Escherichia coli, and other members of the Enterobacteriaceae ${ }^{4}$. The most prevalent ESBLs are included in three groups: TEM, SHV and CTX-M ${ }^{5}$. CTX-M type ESBLs show only $40 \%$ identity to TEM or SHV ESBLs, but they are closely related to $\beta$-lactamases of the Kluyvera spp ${ }^{6}$.

Carbapenems were the drug of choice for the treatment of multidrug resistant gram-negative bacterial infections. Emergence
1,3,4,5 Department of Microbiology, Manipal Hospital, Bengaluru, Karnataka, India

${ }^{2}$ Department of Microbiology, Kasturba Medical College, Mangaluru, Karnataka, India

Corresponding Author: Beena Hosdurg Bhaskar, Department of Laboratory Medicine, Microbiology, Manipal Hospital, Bengaluru, Karnataka, India, e-mail: beenhb@yahoo.co.in

How to cite this article: Beena HB, Shenoy SM, et al. Molecular Characterization of Extended Spectrum $\beta$-lactamase and Carbapenemase Producing Klebsiella pneumoniae from a Tertiary Care Hospital. Indian J of Crit Care Med 2019;23(2):61-66.

Source of support: Nil

Conflict of interest: None

of carbapenem resistant bacteria left limited options in the choice of antibiotics to treat the infections caused by them ${ }^{7}$. These bacteria have the potential to spread rapidly within the hospital environment and also across the continents ${ }^{8}$. Resistance to carbapenem is mostly due to production of enzymes-carbapenemases that hydrolyze carbapenems and other $\beta$-lactams. Acquired carbapenemases belong to group A (IMI, NMC, SME GES, and Klebsiella pneumoniae carbapenemase (KPC), group B metallo- $\beta$-lactamase (MBLs of VIM, IMP, GIM, NDM, SIM, and DIM series), and group D (carbapenem hydrolyzing oxacillinases e.g. OXA $48^{9}$.

NDM-1 producing bacteria are important because the gene encoding this enzyme is located on a transmissible plasmid (of varying size). It is also associated with other resistant determinants

(0) The Author(s). 2019 Open Access This article is distributed under the terms of the Creative Commons Attribution 4.0 International License (http://creativecommons. org/licenses/by/4.0/), which permits unrestricted use, distribution, and non-commercial reproduction in any medium, provided you give appropriate credit to the original author(s) and the source, provide a link to the Creative Commons license, and indicate if changes were made. The Creative Commons Public Domain Dedication waiver (http://creativecommons.org/publicdomain/zero/1.0/) applies to the data made available in this article, unless otherwise stated. 
leading to extensive drug resistance which is usually exhibited by a majority of the NDM-1 producing enterobacteriaceae leaving only a few therapeutic options.

Therefore NDM-1 producing organisms are also named as "Super bugs". NDM-1 was first identified and reported in 2009 in Klebsiellae pneumoniae and Escherichia coli. It was isolated from Swedish patient of Indian origin who was previously hospitalized in New Delhi, India ${ }^{10}$. Indian investigators identified E. coli and Klebsiella species containing the gene for NDM-1 in multiple geographic regions in India, Pakistan and Bangladesh ${ }^{11}$.

The objective of this study was molecular characterization of the enzymatic mechanisms of resistance to $\beta$-lactam antibiotics in K. pneumoniae. A multiplex polymerase chain reaction (PCR) was setup for the detection of CTX-M, TEM and SHV genes. The reduced susceptibility to carbapenems by disk diffusion test prompted us to determine the molecular assay on these isolates to detect KPC and NDM-1 genes and also to analyze coexistence of AmpC producers among Klebsiella pneumoniae isolates at a tertiary care hospital.

\section{Materials and Methods}

\section{Bacterial Isolates}

A total of 250 nonrepetitive clinical isolates of K.pneumoniae were recovered over a period of one year (2014-2015) from our intensive care units (ICUs), i.e. medical ICU (MICU), neurosurgery ICU (NSICU), intensive thoracic unit (ITU), neonatal ICU (NICU), pediatric ICU (PICU), coronary care unit (CCU), and renal ICU (RICU). These isolates obtained from various clinical samples such as endotracheal aspirate ( $n=103)$, blood $(n=56)$, urine $(n=31)$, pus $(n=22)$, sputum $(n=3)$, bronchoalveolar lavage $(n=11)$, central nervous catheter tips ( $n=13)$, and sterile body fluids ( $n=11)$. The present study was carried out in a tertiary care hospital of Karnataka, South India, with bed strength of 618 .

\section{Antimicrobial Susceptibility Testing}

The susceptibilities of the different $\beta$-lactam and non- $\beta$-lactam antibiotics were tested and the results were interpreted as per the Clinical and Laboratory Standards Institute guidelines ${ }^{12}$. Escherichia coli ATCC 25,922 was used as a quality control. The antibiotics were procured from Hi Media, Mumbai, Maharashtra, India.

\section{Minimum Inhibitory Concentration (MIC)}

MIC determination was performed for all the isolates by agar dilution method $(\mathrm{CLSI})^{12}$. Among NDM-1 producers, the MIC of meropenem and colistin ranged between $4-32 \mu \mathrm{g} / \mathrm{mL}$ and $0.25-256$ $\mu \mathrm{g} / \mathrm{mL}$, respectively.

\section{Detection of Extended Spectrum $\beta$-lactamase Producers}

Isolates resistant or intermediately resistant to aztreonam, cefotaxime and/or ceftazidime were phenotypically detected for the presence of ESBL by the Double Disk Synergy test using cefotaxime $(30 \mu \mathrm{g})$ and cefotaxime + clavulanic acid $(30 / 10 \mu \mathrm{g})$ and $(30 / 10 \mu \mathrm{g})^{12}$. K. pneumoniae ATCC 700603 was used as the ESBL positive control and E. coli ATCC 25,922 was used as the negative control.

\section{Detection of Carbapenemase Producers}

Isolates resistant or intermediately resistant to imipenem, ertapenem and/or meropenem were phenotypically detected for the production of carbapenemases by the modified Hodge test using ertapenem $(10 \mu \mathrm{g})$ as an indicator disc and by comparing the zone diameter surrounding ertapenem discs supplemented with and without $0.5 \mathrm{M}$ EDTA $(750 \mu \mathrm{g})$, an increase of zone diameter by $\geq 4 \mathrm{~mm}$ suggested the production of metallocarbapenemase ${ }^{13}$.

MICs of meropenem and colistin (Sigma-Aldrich Corporation, St. Louis, US) were determined by the agar dilution method according to the guidelines from the $\mathrm{CLSI}^{12}$. The colistin breakpoint was evaluated using breakpoints for Enterobacteriaceae recommended by the European Committee on Antibiotic Susceptibility Testing. (Resistant: $>2 \mu \mathrm{g} / \mathrm{mL}$; sensitive: $\leq 2 \mu \mathrm{g} / \mathrm{mL}$ ). K. pneumoniae ATCC 700603 was used as a quality control.

\section{Phenotypic Detection of AmpC Production}

AmpC phenotype was detected by means of combined disc method using cefoxitin disc ( $30 \mu \mathrm{g}$ ) (Hi-media Laboratories, Mumbai), alone and in combination with $400 \mu \mathrm{g}$ of phenylboronic acid (BA) (SigmaAldrich, Fluka, China) ${ }^{14}$.

\section{DNA Extraction and Amplification}

Total DNA was extracted as described by Lee $\mathrm{J} \mathrm{H}^{15}$. The extracted DNA was subjected to multiplex PCR for the detection of ESBLs and uniplex PCR for NDM and KPC genes ${ }^{16,17,18}$.

The Universal primers used for PCR amplification were as follows:

- bla $a_{\text {CTXM }}$ F-5'-CGCTTTGCGATGCGAG-3'

- bla $a_{\text {CTXM }}$ R-5'-ACCGCGATATCGTTG-3'

- bla $a_{\text {TEM }}$ F-5'-CATTTCCGTGTCGCCCTTATTC-3'

- $\quad b l a_{\mathrm{TEM}} \mathrm{R}-5^{\prime}$-CGTTCATCCATAGTTGCCTGAC-3'

- bla $a_{\mathrm{SHV}}$ F-5'- GTTCATCCATAGTTGCCTGAC-3'

- $b a_{\mathrm{SHV}} \mathrm{R}-5^{\prime}-\mathrm{AGCCGCTTGAGCAAATTAAAC-3^{ \prime }}$

- $b l a_{\mathrm{NDM}}$ F-5'- GGTTTGGCGATCTGGTTTTC-3'

- bla $a_{\text {NDM }}$ R-5'- GAATGGCTCATCACGATC-3'

- bla $a_{\mathrm{KPC}}$ F-5'- ATGTCACTGTATCGCCGTCT-3'

- $b / a_{\mathrm{KPC}} \mathrm{R}-5^{\prime}-\mathrm{TTTTCAGAGCCTTACTGCCC-3^{ \prime }}$

\section{Results}

A total of $250 \mathrm{~K}$. pneumoniae were isolated from different clinical samples. The distribution of these isolates in clinical specimens and in ICUs is shown in Graphs 1 and 2.

Out of $250 \mathrm{~K}$. pneumoniae isolates, 210 were screened positive for ESBL producers. The distribution of bla genes among ESBL positive K. pneumoniae and the gel picture showing the multiplex PCR are given in Graphs 3 and 4.

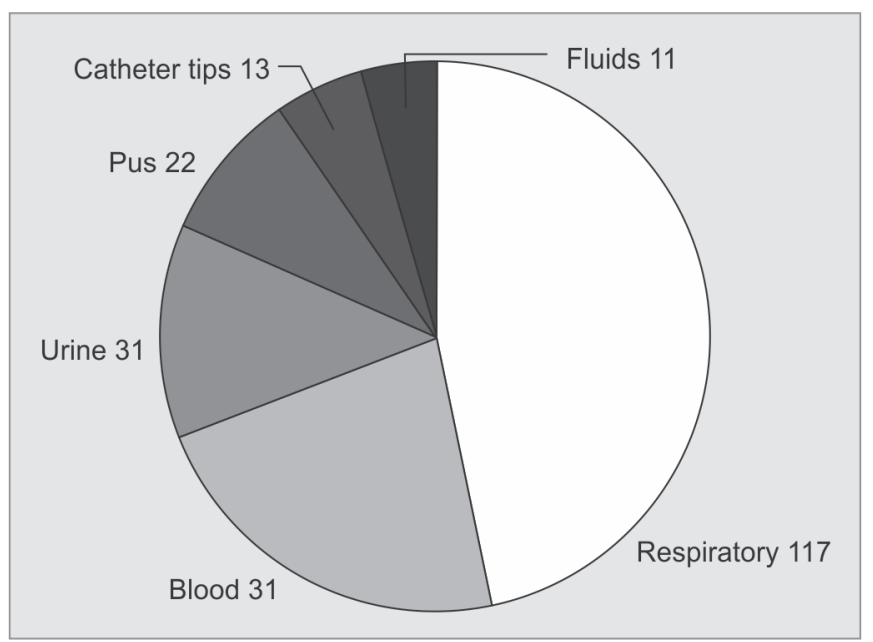

Graph 1: Clinical sources of ESBL producing K. pneumoniae isolates 


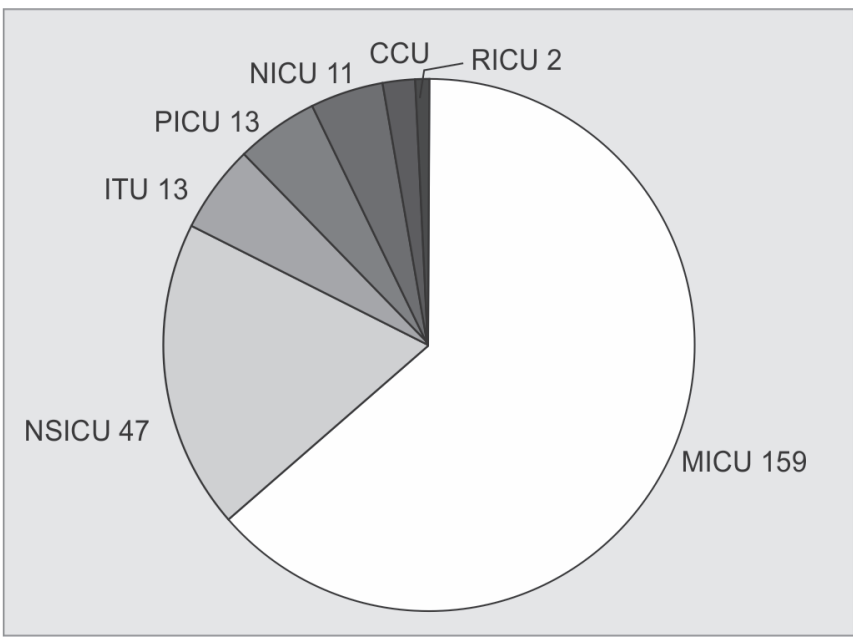

Graph 2: Distribution of K. pneumoniae in ICUs

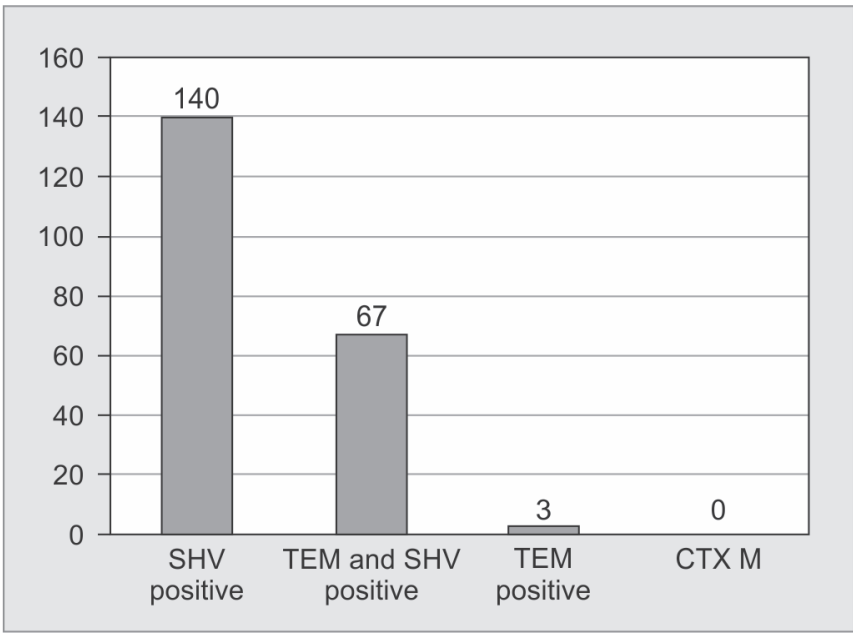

Graph 3: Distribution of bla genes among ESBL positive K. pneumoniae

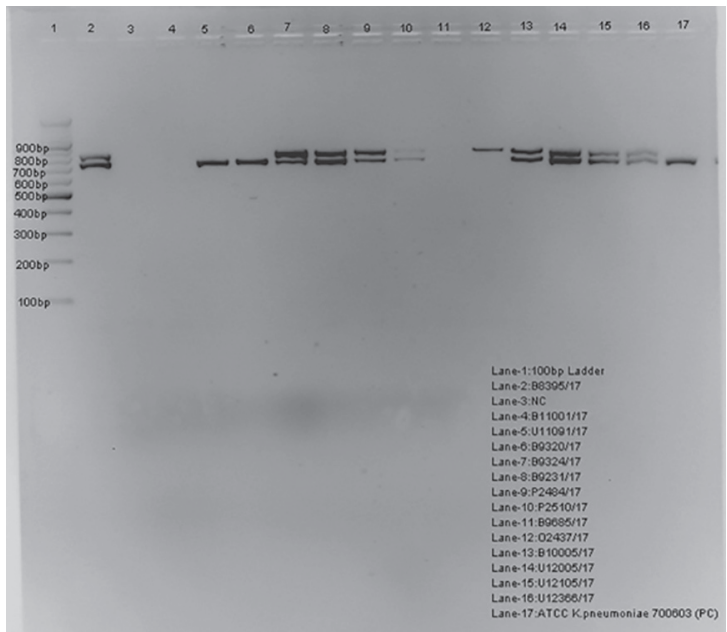

Graph 4: Agarose gel showing multiplex PCR amplified product of bla $a_{\mathrm{TEM}}$ and $b / a_{\mathrm{SHV}}$ genes

Lane-1 = $100 \mathrm{bp}$ ladder (Thermo scientific, Made in (EU) Lithuania). Lane $-2,5$ and $14=b l a_{\mathrm{TEM}}$, bla $a_{\mathrm{SHV}}$ positive amplicons (800 bp and 713 bp, respectively). Lane-3: Negative control (no template DNA added).
The susceptibility of ESBL producers found to be, amikacin (42\%), netilimicin (36.5\%), imipenem (35\%), meropenem (34\%), ertapenem (34\%), cefaperazone/sulbactum (31\%) and piperacillin/ tazobactum (28\%), respectively.

Out of $250 \mathrm{~K}$. pneumoniae isolates, a total of $165(66 \%)$ isolates were carbapenem resistant. The distribution of carbapenem resistant $K$. pneumoniae isolates in clinical samples and in ICUs is given in Graphs 5 and 6.

\section{Prevalence of Carbapenemase Activity Based on Phenotypic tests}

The phenotypic detection of carbapenemase production was performed as mentioned in Table 1.

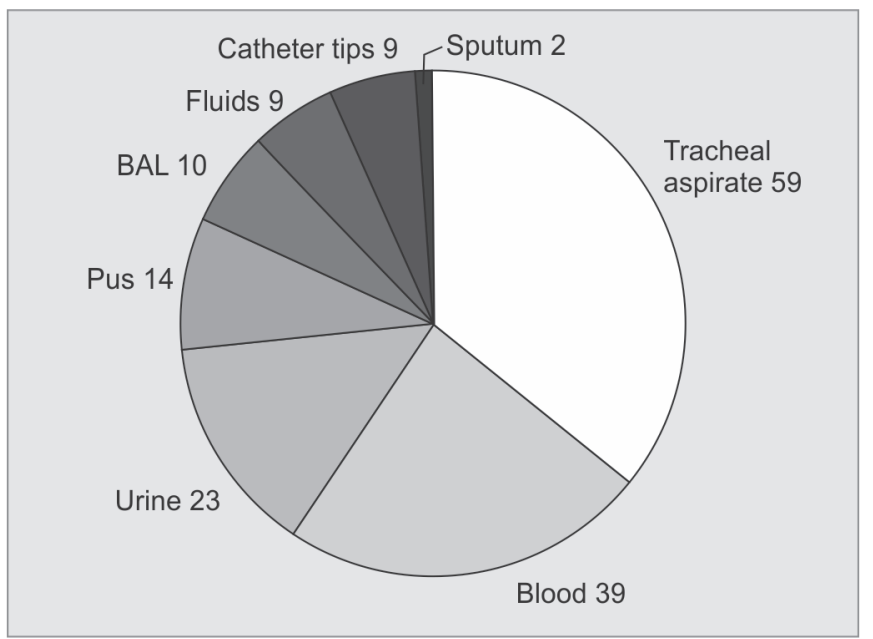

Graph 5: Distribution of carbapenem resistant K. pneumoniae in clinical samples

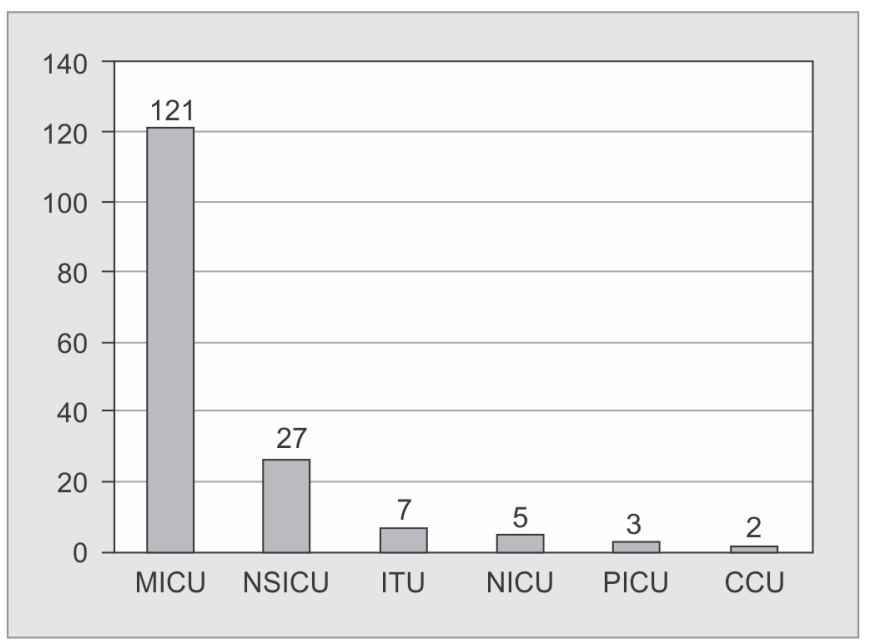

Graph 6: Distribution of carbapenem resistant Klebsiella pneumoniae in ICU's

Table 1: Carbapenem resistant Klebsiella pneumoniae isolates positive for phenotypic tests

\begin{tabular}{lll}
\hline Test & Number & Percentage (\%) \\
\hline MHT & 96 & 58.2 \\
EDTA $^{*}$ & 28 & 16.9 \\
AmpC & 6 & 3.6 \\
Total & 130 & 78.7 \\
\hline
\end{tabular}




\section{Prevalence and Distribution of Carbapenemase Genes}

Plasmid DNA was extracted from all the screened positive isolates ${ }^{15}$. The extracted plasmid DNA of each isolate was subjected to PCR detection of the $b / a_{\mathrm{NDM}-1}$ and $b / a_{\mathrm{KPC}}$ genes by using target specific primers ${ }^{17,18}$, which is given in Table $2^{17,18}$.

Agarose gel showing PCR amplified product of bla $a_{\mathrm{NDM}-1}$ genes and susceptibility pattern of $\boldsymbol{b} / \boldsymbol{a}_{\mathrm{NDM}-1}$ isolates is shown in Figure 1 and Graph 7.

\section{Discussion}

Klebsiella has been associated with different types of infections and one of the important aspects of Klebsiella associated infections is the emergence of multidrug resistant strains particularly those involved in nosocomial diseases.

Table 2: Distribution of carbapenemase encoding genes in carbapenem resistant $K$. pneumoniae isolates

\begin{tabular}{lll}
\hline bla Encoding Genes & Number & Percentage (\%) \\
\hline bla $_{\mathrm{NDM}-1}$ & 16 & 9.7 \\
$b l a_{\mathrm{KPC}}$ & nil & 0 \\
\hline
\end{tabular}

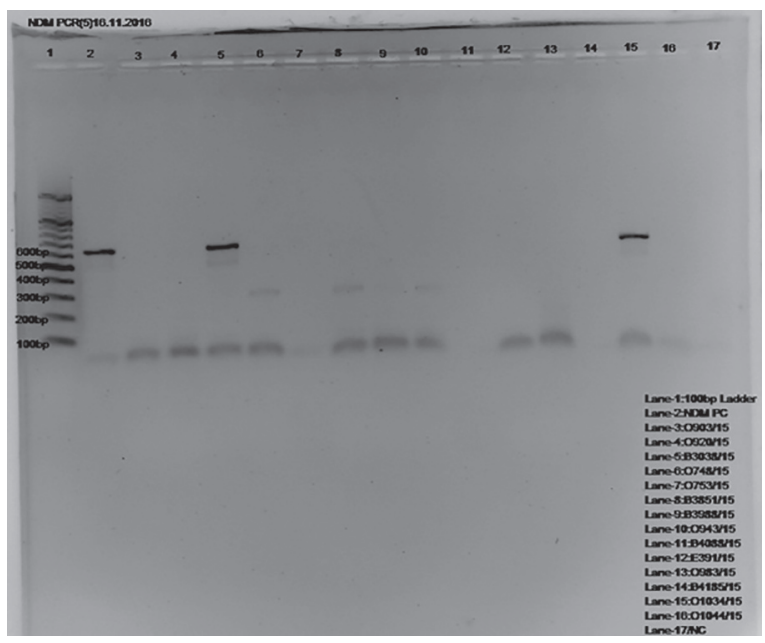

Fig. 1: Agarose gel showing PCR amplified product of $b / a_{\mathrm{NDM}-1}$ gene. Lane- $1=100$ bp ladder (Thermo scientific, Made in (EU) Lithuania). Lane $-2,5$ and $15=b l a_{\mathrm{NDM}-1}$ postive amplicons (621 bp). Lane-3: Negative control (no template DNA added)

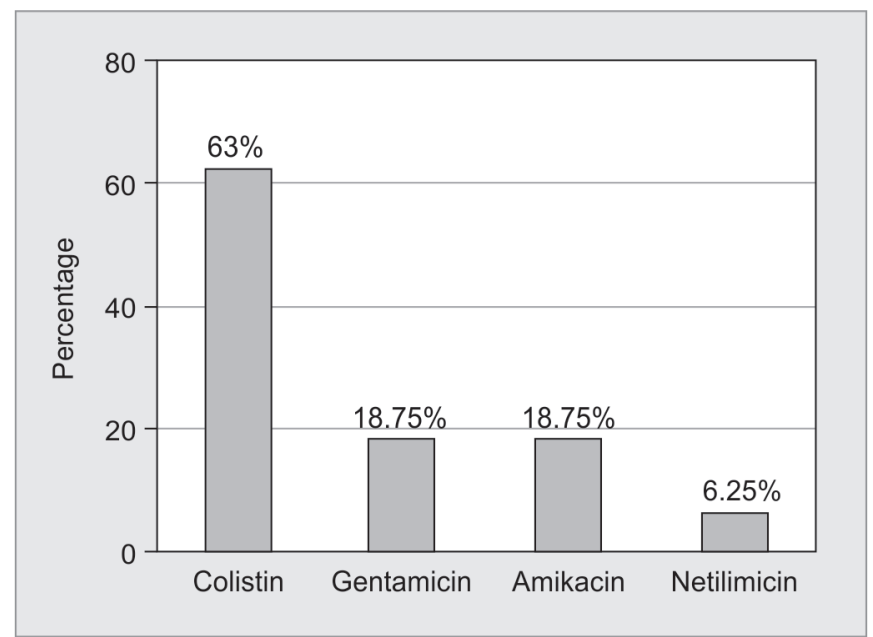

Graph 7: Susceptibility pattern of $b / a_{\mathrm{NDM}-1}$ isolates by disk diffusion
In our study, the prevalence of ESBL production was $84 \%$ which was higher when compared to other studies. Sharma et al found $67.04 \%$, Hoda Hassan et al 66.7\%, and Mohammed et al 30\% ${ }^{19,20,21}$ It was similar to a study by Feizabadi et al, with $72.1 \%$ prevalence ${ }^{22}$. None of the isolates were positive for CTX M gene in our study.

Among these $210 \mathrm{ESBL}$ positive K. pneumoniae isolates, 14 (7.8\%) of them found to be AmpC producers. A study by Hemalatha et al found 7 (9.2\%) isolates positive for $\mathrm{AmpC}^{23}$. Two other Indian studies reported $8 \%$ and $43 \% \mathrm{AmpC}$ producers ${ }^{24,25}$.

K. pneumoniae have acquired carbapenemases, the enzymes capable of breaking down most $\beta$-lactams including carbapenems, and confer resistance to these drugs. Reports indicate that carbapenemase producing enterobacteriaceae isolates seem to be increasing in number in the last few years. In our study, we found $66 \%$ of the isolates were carbapenemase producers. Gupta et al., from All India Institute of Medical Sciences, New Delhi in 2006 found that resistance to meropenem was $6.9 \%{ }^{26}$. Nagaraj et al observed $75 \%$ of the $K$. pneumoniae isolates were carbapenem resistant in their study ${ }^{8}$. Aseem et al stated that $35.3 \%$ of the isolates in their study were resistant to carbapenems ${ }^{27}$.

Genotypic analysis of these carbapenem resistant isolates revealed the prevalence of the $b / a_{\mathrm{NDM}-1}$ as $9.7 \%$ and none of the isolates were positive for $b / a_{\mathrm{KPC}}$ in our study. The epidemiology of K. pneumoniae producing KPCs varies geographically. Globally, the highest rate of carbapenem resistance has been reported in Greece with $68 \%$ resistance (KPC, OXA-48-like, NDM) followed by India (NDM, OXA-48-like, KPC) and eastern Mediterranean regions (NDM, OXA-48-like) with 54\% resistance. USA, China (KPC, NDM, OXA -48-like) and Africa (OXA-48-like, NDM) have low resistance rates with 11,11 and $4 \%$ respectively ${ }^{28}$

The predominant enzymatic mechanism of resistance in Europe is KPC followed by OXA-48-like and NDM, while in USA; it is KPC followed by NDM and minimal due to OXA-48-like ${ }^{29}$. Several studies in Spain showed that most carbapenemase-producing $K$. pneumoniae harbored OXA-48-like or class B carbapenemases, and that of KPC-producing K. pneumoniae was very low $(2-3 \%)^{30,31}$.

Lascos et al. reported $34.8 \%$ prevalence of $b / a_{\mathrm{KPC}}$ in CRE and Aseem et al. observed only $3.7 \%$ of bla $a_{\mathrm{KPC}}$ out of $35.2 \%$ carbapenem resistant Klebsiella pneumoniae isolates ${ }^{8,30}$.

However, in our study, none of the isolates were positive for bla $a_{\mathrm{KPC}}$. This correlates with findings of Nagaraj et al. who has observed $75 \%$ of $b / a_{\mathrm{NDM}}$ but not detected $b / a_{\mathrm{KPC}}$ from any of the carbapenem resistant isolates ${ }^{8}$. A study from Vellore, by Veeraraghavan et al showed the coexpression of $b / a_{\mathrm{NDM}}$ and $b / a_{\mathrm{OXA} 48}$ in $28 \%, b / a_{\mathrm{NDM}} 19 \%, b / a_{\mathrm{OXA} 48}$ in $13 \%$ and $b l a_{\mathrm{KPC}}$ was absent among the carbapenem resistant isolates ${ }^{33}$. The endemic spread of NDMproducing $K$. pneumoniae has also been reported in the UK, which has close relationships with India and Pakistan ${ }^{32}$.

The Balkan states, the Arabian Peninsula, and North African countries have also been recently considered as an additional reservoir of NDM producers ${ }^{32-35}$. In the Arabian Peninsula, NDM-1 was the most frequently encountered carbapenemase $46.5 \%$, followed by OXA-48-like carbapenemases $32.5 \%{ }^{36}$. In India, a study by Deshpande et al, reported NDM-1 was the most common carbapenemase type detected and accounted for $75.2 \%$ of the carbapenemase-producing isolates ${ }^{37}$. In another study, an incidence of bla $a_{\mathrm{NDM}-1}$ in a single K.pneumoniae isolate was reported from a surgical site infection ${ }^{38}$. In our study we found $9.7 \%$ of NDM-1 which is similar to a study from tertiary care hospital of Northeast India where they found $8.67 \%$ of NDM pocessing Klebsiella pneumoniae isolates ${ }^{39}$. 
It was observed that the coexistence of bla gene along with NDM producers found to be $69 \%$ and $50 \%$ of SHV and TEM respectively. Previous studies from India have reported the coexistence of bla gene along with NDM producers ${ }^{39-41}$. Similar studies from abroad also showed the presence of bla genes along with NDM producers ${ }^{42,43}$.

We found $78.7 \%$ of the CRKP were positive for the production of one or more carbapenemase mechanisms phenotypically. It has been observed that among the screened CRKP, 9.7\% isolates found to harbor bla $a_{\mathrm{NDM}-1}$ gene. Rest of the CRKP isolates did not possess any of the resistant mechanisms tested. The important contributing factors leading to carbapenem resistance in these isolates might be due to the hyper production of ESBL, or other enzymatic mechanisms of carbapenem resistance like, IMP, VIM, and OXA, porin loss or efflux pumps ${ }^{44,45}$.

\section{Conclusion}

In conclusion, carbapenem resistant $K$. pneumoniae have been considered as one of the greatest threats to the global health care in this century. The prevalence of KPC gene i.e., bla $a_{\mathrm{KPC}}$ in carbapenem resistant isolates from our geographical area (South Western India) seems to be very less. Pathogens that produce carbapenemases along with an ESBL or AmpC $\beta$-lactamases are particularly challenging for clinicians and are a major threat worldwide. Moreover, the widespread dissemination of the new NDM-1 metallo- $\beta$-lactamase requires particular attention as the genetic background demonstrates extreme mobility and versatility. The dissemination of such plasmids between different clinically important bacterial species may lead to serious public health issues, as K. pneumoniae accounts for one of the important bacterial species in the dissemination of antibiotic resistance genes; particularly in hospital environments. Therefore, the early detection of the $b / a_{\mathrm{NDM}-1}$ possessing K. pneumoniae isolates is necessary with any reduced susceptibility to the carbapenems. Timely intervention in the form of rapid detection, good infection control practices and judicious use of antibiotics will ensure that the spread of drug resistance among bacteria can be kept under control.

\section{References}

1. Giske CG, Monnet DL, et al. ReAct-Action on antibiotic resistance. Clinical and economic impact of common multidrug-resistant gramnegative bacilli. Antimicrob Agents Chemother. 2008;52:813-821.

2. Perez F, Endimiani $A$, et al. The continuing challenge of ESBLs. Curr Opin in Pharmacol.2007;7:459-469.

3. Johann DD, Pitout DK, et al. Extended spectrum beta lactamases producing Enterobacteriaceae: an emerging public health concern. Lancet Infect Dies 2008;8:159-166.

4. Kang Cl, Kim SH, et al. Bloodstream infections due to extendedspectrum beta-lactamase producing Escherichia coli and Klebsiella pneumoniae:risk factors for mortality and treatment outcome, with special emphasis on antimicrobial therapy. Antimicrob Agents Chemother 2004; 48: 4574-4581.

5. Lin CF, Hsu SK, et al. Genotypic detection and molecular epidemiology of extended-spectrum beta-lactamase-producing Escherichia coli and Klebsiella pneumoniae in a regional hospital in central Taiwan. J Med Microbiol. 2010;59: 665-671.

6. Baby Padmini S, Appala Raju B, et al. Detection of Enterobacteriaceae producing CTX-M extended spectrum beta-lactamases from a tertiary care hospital in south India. Indian J Med Microbiol.2008; 26:163-166.

7. Walsh TR. Emerging carbapenemases.A Global perspective.Int J Antimicrob agents 2010;36: S8-14.
8. Nagaraj S, Chandran SP, et al. Carbapenem resistance among Escherichia coli and Klebsiellae pneumoniae in a tertiary care hospital, South India. Indian J Med Microbiol 2012; 30:93-95.

9. Nordmann P, Naas T, et al. Global spread of carbapenemaseproducing Enterobacteriaceae.Emerg Infect Dis.2011; 17:1791-1798.

10. Yong D, Toleman MA, et al. Characterization of a new metallo-betalactamase gene, blaNDM-1, and a novel erythromycin esterase gene which was carried on a unique genetic structure in Klebsiella pneumoniae, sequence type 14 from India. Antimicrob Agents Chemother 2009; 53:5046-5054.

11. Kumaraswamy KK, Toleman MA, et al. Emergence of a new antibiotic resistance mechanism in India, Pakistan, and the UK: a molecular, biological, and epidemiological study. Lancet Infect Dis 2010; 10:597602.

12. Clinical and Laboratory Standards Institute. Performance standards for antimicrobial susceptibility testing, Twenty-First informational supplement. CLSI document M100-S21. Wayne, PA: CLSI; 2011.

13. Yong $D$, Lee $K$, et al. Imipenem-EDTA disk method or differentiation of metallo- $\beta$-lactamase- producing clinical isolates of Pseudomonas spp and Acinetobacter spp. J Clin Microbiol 2002; 40:3798-3801.

14. Coudron PE. Inhibitor based methods for detection of plasmidmediated AmpC beta-lactamases in Klebsiella spp., Escherichia coli, and Proteus mirabilis. J Clin Microbiol 2005; 43:4163-4167.

15. Lee J H. Methicillin (Oxacillin)-Resistant Staphylococcus aureus strains isolated from Major food animals and their potential transfer to Humans. Appl Environ Microbiol 2003; 69:6489-6494.

16. Manoharan A, Premlatha K, et al. SARI study group India. Correlation of TEM, SHV and CTX-M extended-spectrum beta lactamases among Enterobacteriaceae with their in vitro antimicrobial susceptibility. Indian J Med Microbiol 2011; 29:161-164.

17. Nordmann $P$, Poirel $L$, et al. How to detect NDM-1 producers? J.Clin. Microbiol.2011; 49:718-721.

18. S. Vered, SR Keren, et al. Evaluation of PCR-Based testing for surveillance of KPC-producing Carbapenem-Resistant members of the Enterobacteriaceae family. J. Clin. Microbiol 2009; 47:3261-3265

19. Sharma M, Pathak $S$, et al. Prevalence and antibiogram of Extended Spectrum $\beta$-Lactamase (ESBL) producing Gram negative bacilli and further molecular characterization of ESBL producing Escherichia coli and Klebsiella spp. J Clin Diagn Res 2013;7: 173-177

20. Hoda Hassan, B.A. Molecular characterization of extended-spectrum beta-lactamase. Producing Enterobacteriaceae in a Saudi Arabian tertiary hospital. J Infect Dev Ctries 2014;8: 282-288.

21. Mohammed Y, Gadzama GB, et al. Characterization of ExtendedSpectrum Beta-lactamase from Escherichia coli and Klebsiella Species from North Eastern Nigeria Journal of Clinical and Diagnostic Research 2016;10:7-10.

22. Feizabadi MM, Raji N, et al. Distribution of bla(TEM), bla(SHV), bla(CTX-M) genes among clinical isolates of Klebsiella pneumoniae at Labbafinejad Hospital, Tehran, Iran.Iran Microb Drug Resist 2013; 16:49-53

23. Hemalatha V, Padma M, et al. Detection of Amp C beta lactamases production in Escherichia coli and Klebsiella by an inhibitor based method. Indian J Med Res 2007;126:220-223.

24. Manchanda V, Singh NP, et al. Molecular epidemiology of clinical isolates of AmpC producing Klebsiella pneumoniae. Indian J Med Microbiol 2006; 24:177-181.

25. Black JA, Thomson KS, et al. Evaluation of beta-lactamase inhibitors in disk tests for detection of plasmid-mediated AmpC beta-lactamases in well characterized clinical strains of Klebsiella spp. J Clin Microbiol 2005;43:4168-4171.

26. Gupta E, Mohanty S, et al. Emerging resistance to carbapenems in a tertiary care hospital in north India. Indian J Med Res. 2006;124:95-8.

27. Aseem R, Shenoy S, et al. Approach to carbapenemase detection in Klebsiella pneumoniae in routine diagnostic laboratories. J Clin Diagn Res 2016; 10:24-27

28. Oteo J, Saez D, et al. Carbapenemase-producing enterobacteriaceae in Spain in 2012. Antimicrob.Agents Chemother 2013;57:6344-47. 
29. Palacios-Baena, ZR, et al. Comprehensive clinical and epidemiological assessment of colonisation and infection due to carbapenemaseproducing Enterobacteriaceae in Spain. J. Infect. 2016;72:152-60.

30. Lascols C, Hackel M, et al. Increasing prevalence and dissemination of NDM-1 metallo-beta-lactamase in India: data from the SMART study (2009). J Antimicrob Chemother 2011;66:1992-7.

31. Veeraraghavan B, Shankar C, et al. Carbapenem resistant Klebsiella pneumoniae isolated from bloodstream infection: Indian experience. Pathogens and Global Health. 2017;111:240-46.

32. Voulgari E, Gartzonika C, et al. The balkan region:NDM-1-producing Klebsiella pneumoniaeST11clonal strain causing outbreaks in Greece. J. Antimicrob. Chemother 2014;69: 2091-97.

33. Dortet L, Brechard L, et al. Rapid detection of carbapenemaseproducing Enterobacteriaceae from blood cultures. Clin. Microbiol. Infect 2014;20:340-44.

34. Sonnevend A, Ghazawi A, et al. Characterization of KPC-type carbapenemase-producing Klebsiella pneumoniae strains isolated in the Arabian Peninsula. J. Antimicrob. Chemother 2015;70:1592-93.

35. Kazi M, Drego L, et al. Molecular characterization of carbapenemresistant Enterobacteriaceae in tertiary care laboratory in Mumbai. Eur. J.Clin.Microbiol.Infect.Dis 2015;34:467-72.

36. Sarma JB, Bhattacharya PK, et al. Multidrug-resistant Enterobacteriaceae which include metallo- $\beta$-lactamase producers are the predominant pathogens of healthcare-associated infections in an Indian teaching hospital. Indian J Med Microbiol 2011;29:22-7.

37. Deshpande $\mathrm{P}$, Rodrigues $C$, et al. New Delhi Metallo-beta lactamase (NDM-1) in Enterobacteriaceae: the treatment options with the carbapenems are compromised. J Assoc Physicians India 2010;58:147-9.
38. Chacko B, Peter JV, et al. The New Delhi Metallo-Beta-Lactamases: Their origins and implications for the intensivists. J Global Infect Dis 2011;3:319.

39. Ahmed N, Khalid S, et al. Occurrence of blaNDM Variants Among Enterobacteriaceae From a Neonatal Intensive Care Unit in a Northern India Hospital. Front Microbiol 2018;9:1-10.

40. Samuelsen $\varnothing$, Thilesen CM, et al. Identification of NDM-1- producing Enterobacteriaceae in Norway. J Antimicrob Chemother 2011; 66:670-2.

41. Poirel L, Revathi G, et al. Detection of NDM- 1-producing Klebsiella pneumoniae in Kenya. Antimicrob Agents Chemother 2011;55:934-36.

42. Kanj SS, Kanafani ZA. Current concepts in the antimicrobial therapy against resistant gram-negative organisms: extended-spectrum beta-lactamase-producing Enterobacteriaceae, carbapenemresistant Enterobacteriaceae, and multidrug-resistant Pseudomonas aeruginosa. Mayo Clin Proc 2011;86:250-59.

43. Jeremiah S, Balaji $\mathrm{V}$, et al. A possible alternative to the error prone modified Hodge test to correctly identify the carbapenemase producing Gram-negative bacteria. Indian J Med Microbiol. 2014;32(4):414.

44. Shanthi M, Sekar U, et al. OXA-181 beta lactamase is not a major mediator of carbapenem resistance in Enterobacteriaceae.

45. Li H, Zhang J, et al. Molecular characteristics of carbapenemaseproducing Enterobacteriaceaein China from 2008 to 2 011:predominance of KPC-2enzyme. Diagn.Microbiol.Infect Dis 2013;78:63-65. 